JOURNAL OF OPTIMIZATION, DIFFERENTIAL EQUATIONS AND THEIR APPLICATIONS (JODEA) Volume 26, Issue 2, December 2018, pp. 1-12, DOI 10.15421/141807

ISSN (print) 2617-0108

ISSN (on-line) $\mathrm{xxxx}-\mathrm{xxxx}$

\title{
UNIFORM ATTRACTORS FOR VANISHING VISCOSITY APPROXIMATIONS OF NON-AUTONOMOUS COMPLEX FLOWS
}

\author{
Nataliia V. Gorban, Oleksiy V. Kapustyan† Pavlo O. Kasyanov; \\ Olha V. Khomenko, Liliia S. Paliichuk, José Valero, \\ Michael Z. Zgurovsky**
}

\begin{abstract}
In this paper we prove the existence of uniform global attractors in the strong topology of the phase space for semiflows generated by vanishing viscosity approximations of some class of non-autonomous complex fluids.
\end{abstract}

Key words: non-Newtonian fluids, parabolic equations, global attractors, infinite-dimensional dynamical systems..

2010 Mathematics Subject Classification: 35B40, 35B41, 35K55, 37B25.

\section{Communicated by Prof. O. M. Stanzhytskyi}

\section{Introduction}

In this paper we consider a non-autonomous evolution problem which appears in the investigation of the model of concentrated suspensions (proposed by Hebraud and Lequex [12]) with non-autonomous coefficients. More precisely, the unknown function $p(x, t)$, representing probability density, satisfies the following equation:

$$
\frac{\partial p}{\partial t}=-b(t) \frac{\partial p}{\partial x}+D(p) \frac{\partial^{2} p}{\partial x^{2}}-\chi_{\mathbb{R} \backslash[-1,1]}(x) p+\frac{D(p)}{\alpha} \delta_{0}(x),
$$

where $\alpha>0$ is a parameter, $\chi_{\mathbb{R} \backslash[-1,1]}$ is the characteristic function of the open set $\mathbb{R} \backslash[-1,1], \delta_{0}$ is the Dirac delta function with support at the origin,

$$
D(f)=\alpha \int_{|x|>1} f(x) d x,
$$

\footnotetext{
*Institute for Applied System Analysis, National Technical University of Ukraine "Igor Sikorsky Kyiv Polytechnic Institute", Peremogy ave., 37, build, 35, 03056, Kyiv, Ukraine, nata_gorban@i.ua

${ }^{\dagger}$ Taras Shevchenko National University of Kyiv, Kyiv, Ukraine, alexkap@univ.kiev.ua

${ }^{\ddagger}$ Institute for Applied System Analysis, National Technical University of Ukraine "Igor Sikorsky Kyiv Polytechnic Institute", Peremogy ave., 37, build, 35, 03056, Kyiv, Ukraine, kasyanov@i.ua

${ }^{\S}$ Institute for Applied System Analysis, National Technical University of Ukraine "Igor Sikorsky Kyiv Polytechnic Institute", Peremogy ave., 37, build, 35, 03056, Kyiv, Ukraine, olgkhomenko@ukr.net

"Institute for Applied System Analysis, National Technical University of Ukraine "Igor Sikorsky Kyiv Polytechnic Institute", Peremogy ave., 37, build, 35, 03056, Kyiv, Ukraine, lili262808@gmail.com

"Universidad Miguel Hernandez de Elche, Centro de Investigación Operativa, Avda. Universidad s/n, 03202-Elche (Alicante), Spain, jvalero@umh.es

** National Technical University of Ukraine "Igor Sikorsky Kyiv Polytechnic Institute"Peremogy ave., 37, 03056, Kyiv, Ukraine, mzz@kpi.ua

(C) N.V. Gorban at all, 2018 .
} 
and the function $b(t)$ is assumed to be non-autonomous. Moreover, mechanical background of the model requires boundedness with respect to the time of the average stress function

$$
\tau(t)=\int_{\mathbb{R}} x p(t, x) d x .
$$

Existence and uniqueness results for such model were proved in [4]. The theory of global attractors was applied first for (1.1) in Amigó et al. [1], where the existence of global unbounded attractors with respect to the weak topology was proved for the case $b(t) \equiv 0$. Numerical aspects were investigated in $[2,13]$. The key point in $[4,13]$ was the analysis of the so-called vanishing viscosity approximation system, where the diffusion coefficient was everywhere positive. In $[3,5-10,14-$ $22]$ the existence of global attractor in the strong topology of the phase space for $\mathrm{m}$-semiflow generated by vanishing viscosity approximation was proved. Only autonomous (i.e. $b(t) \equiv$ const) case was considered. In the present paper we extend results from [14] to much more general non-autonomous case, using the uniform global attractor approach [11,23-26].

\section{Setting of the problem and preliminaries}

Let $\alpha>0$ be a positive constant, $0 \leq \varepsilon \ll 1$ be a small parameter, and $b: \mathbb{R}_{+} \rightarrow \mathbb{R}$ be a measurable function. Consider the following evolution problem with non-degenerate diffusion:

$$
\begin{gathered}
\frac{\partial p}{\partial t}=-b(t) \frac{\partial p}{\partial x}+(D(p)+\varepsilon) \frac{\partial^{2} p}{\partial x^{2}}-\chi_{\mathbb{R} \backslash[-1,1]}(x) p+\frac{D(p)}{\alpha} \delta_{0}(x), \text { a.e. in } \mathbb{R} \times \mathbb{R}_{+} \\
p(x, t) \geq 0, \text { a.e. in } \mathbb{R} \times \mathbb{R}_{+} \\
\int_{\mathbb{R}} p(x, t) d x=1 \text {, a.e. in } \mathbb{R}_{+} ; \\
\int_{\mathbb{R}}|x| p(x, t) d x<\infty \text {, a.e. in } \mathbb{R} .
\end{gathered}
$$

Suppose that $b$ is an essentially bounded function, that is, there exists a constant $B>0$ such that

$$
|b(t)| \leq B \text { for a.e. } t>0 .
$$

Further we will use the following notation:

$$
L^{p}=L^{p}(\mathbb{R}), \quad H^{1}=H^{1}(\mathbb{R}), \quad H^{-1}=\left(H^{1}\right)^{*},
$$

for each $1 \leq p \leq \infty$. Let $\langle\cdot, \cdot\rangle$ be the pairing on $H^{-1} \times H^{1}$ (on $L^{q} \times L^{p}$ respectively with $p \geq 1$ and $1<q \leq \infty$ such that $\frac{1}{p}+\frac{1}{q}=1$ ) that coincides with the inner product on $L^{2}$, that is,

$$
\langle f, u\rangle=\int_{\mathbb{R}} f(x) u(x) d x,
$$


for each $f \in L^{2}$ and $u \in H^{1}$ (for each $f \in L^{q}$ and $u \in L^{p}$, respectively).

Let $0 \leq \tau<T<\infty$ be arbitrary fixed. A solution of equation (2.1) on a finite time interval $[\tau, T]$ is defined as follows.

Definition 2.1. Let $0<\varepsilon \ll 1$. A function $p \in L^{\infty}\left(\tau, T ; L^{1} \cap L^{2}\right) \cap L^{2}\left(\tau, T ; H^{1}\right)$ with $\frac{\partial p}{\partial t} \in L^{2}\left(\tau, T ; H^{-1}\right)$ is called a (weak) solution of equation $(2.1)$ on $[\tau, T]$, if the equality

$$
\begin{aligned}
& \int_{\tau}^{T}\left(\left\langle\frac{\partial p}{\partial t}, \eta\right\rangle+b(t)\left\langle\frac{\partial p}{\partial x}, \eta\right\rangle+(D(p(\cdot, t))+\varepsilon)\left\langle\frac{\partial p}{\partial x}, \frac{\partial \eta}{\partial x}\right\rangle+\int_{|x|>1} p \cdot \eta d x\right) d t \\
& =\int_{\tau}^{T} \frac{D(p(\cdot, t))}{\alpha}\left\langle\delta_{0}, \eta\right\rangle d t
\end{aligned}
$$

holds for each $\eta \in L^{2}\left(\tau, T ; H^{1}\right)$.

Remark 2.1. We note that the right hand-side of equality (2.6) is equal to

$$
\int_{\tau}^{T} \frac{D(p(t))}{\alpha} \eta(0, t) d t .
$$

Remark 2.2. Let $0<\varepsilon \ll 1$, and $p$ be a solution of equation $(2.1)$ on $[\tau, T]$. Since $p \in L^{2}\left(\tau, T ; H^{1}\right)$ and $\frac{\partial p}{\partial t} \in L^{2}\left(\tau, T ; H^{-1}\right)$, then $p \in C\left([\tau, T] ; L^{2}\right)$, and, therefore, the following initial condition

$$
\left.p\right|_{t=\tau}=p_{\tau}(x), \text { a.e. in } \mathbb{R},
$$

makes sense for $p_{\tau} \in L^{1} \cap L^{2}$.

Let

$$
X:=\left\{p \in L^{2}(\mathbb{R}): \int_{\mathbb{R}}|x||p(x)| d x<\infty\right\},
$$

which is a Banach space with the norm

$$
\|p\|_{X}:=\|p\|_{L^{2}}+\int_{\mathbb{R}}|x||p(x)| d x, \quad p \in X .
$$

Remark 2.3. The embedding $X \subset L^{1} \cap L^{2}$ is continuous. Moreover, $X=\bar{L}^{1} \cap L^{2}$, where

$$
\bar{L}^{1}:=\left\{p \in L^{1}: \int_{\mathbb{R}}|x||p| d x<\infty\right\}
$$

is a Banach space with the following norm:

$$
\|p\|_{\bar{L}^{1}}:=\int_{\mathbb{R}}(1+|x|)|p| d x, \quad p \in \bar{L}^{1} .
$$

We understand condition (2.4) in the sense of the following definition. 
Definition 2.2. The solution $p$ of equation $(2.1)$ on $[\tau, T]$ satisfies condition (2.4) on $[\tau, T]$ if $x p \in L^{\infty}\left(\tau, T ; L^{1}\right)$.

Remark 2.4. Let $p$ be a solution of equation (2.1) on $[\tau, T]$. Then $x p \in L^{\infty}\left(\tau, T ; L^{1}\right)$ if and only if $p \in L^{\infty}(\tau, T ; X)$. Moreover, since $p \in L^{\infty}(0, T ; X), p \in C\left([0, T] ; L^{2}\right)$, and $X \subset L^{2}$, we have that $p \in C\left([0, T] ; X_{w}\right)$.

Let $0<\varepsilon \ll 1$ be arbitrary fixed. Cancès et al. [4, Proposition 2.1] proved that for each $p_{\tau}$ such that

$$
p_{\tau} \in L^{1} \cap L^{\infty}, \quad p_{\tau} \geq 0, \quad \int_{\mathbb{R}} p_{\tau}(x) d x=1, \quad \int_{\mathbb{R}}|x| p_{\tau}(x) d x<\infty,
$$

problem $(2.1)-(2.4),(2.7)$ on $[\tau, T]$ has a unique solution $p$. Moreover,

$$
\begin{aligned}
& p \in L^{\infty}(\mathbb{R} \times(\tau, T)), \sigma p \in L^{\infty}\left(0, T ; L^{1}\right), \\
& p \in C\left([\tau, T] ; L^{2} \cap L^{1}\right), D(p) \in C([\tau, T]),
\end{aligned}
$$

and

$$
\int_{\mathbb{R}} p(t, \sigma) d \sigma=1, p(t) \geq 0 \text { for all } t \geq 0 .
$$

Therefore, the phase space for this problem can be defined as follows:

$$
H:=\operatorname{cl}_{X} E, E:=\left\{p \in X: p \in L^{\infty}, p \geq 0, \int_{\mathbb{R}} p(x) d x=1\right\},
$$

where $\operatorname{cl}_{X}$ is the closure in the space $X$ (see Amigó et al. [1]). The convexity of $E$ implies the equality $H=\operatorname{cl}_{X_{w}} E$.

Remark 2.5. For $0<\varepsilon \ll 1$ it is easy to show that for every $p_{\tau} \in E p \in$ $C\left([\tau, T] ;\left(L^{1} \cap L^{\infty}\right)_{w}\right)$. In particular, we have that $p(t) \in E$ for each $t \in[\tau, T]$. Therefore, for each $p \in H$ the following two conditions hold: (a) $p(x) \geq 0$ for a.e. $x \in \mathbb{R}$, and (b) $\int_{\mathbb{R}} p(x) d x=1$ [1, p. 212]. Moreover, for each $0<\varepsilon \ll 1$, $0 \leq \tau<T<\infty$, and $p_{\tau} \in H$ there exists no more than one solution $p$ of problem (2.1)-(2.3), (2.7) on $[\tau, T]$.

The main goal of the present paper is to show the existence of uniform global attractors in the strong topology of the phase space $H$ for the m-semiflow generated by the non-autonomous problem (2.1)-(2.4).

\section{Existence and properties of solutions}

In this section we provide results from [14] about existence and topological properties of (2.1)-(2.4).

Let $\mathcal{K}_{\tau, \varepsilon}^{+}\left(\mathcal{D}_{\tau, \varepsilon}^{+}\right)$denotes the family of all globally defined solutions of problem (2.1)-(2.3) $((2.1)-(2.4))$ on $[\tau, \infty)$ with $p(\tau) \in H$. By definition, $\mathcal{D}_{\tau, \varepsilon}^{+} \subseteq \mathcal{K}_{\tau, \varepsilon}^{+}$ 
Lemma 3.1. [14, Lemma 3.1] There exists a constant $C>0$ such that, if

$$
0 \leq \varepsilon \ll 1, \tau \geq 0 \text { and } p \in \mathcal{K}_{\tau, \varepsilon}^{+} \text {with } p(\tau) \in H,
$$

then $p \in \mathcal{D}_{\tau, \varepsilon}^{+}$and the following inequality holds:

$$
\|p(t)\|_{\bar{L}^{1}} \leq\|p(\tau)\|_{\bar{L}^{1}} e^{-\frac{1}{2}(t-\tau)}+C,
$$

for each $t \geq \tau$. Moreover, for each $\delta>0$ and a bounded set (in $\left.\bar{L}^{1}\right) K \subset H$ there exist constants $T=T(\delta, K)>0$ and $\bar{k}=\bar{k}(\delta, K)>0$ such that for each $0 \leq \varepsilon \ll 1, \tau \geq 0$, and $p \in \mathcal{K}_{\tau, \varepsilon}^{+}$with $p(\tau) \in K$ the following inequality holds:

$$
\int_{|x|>2 k} p(x, t)|x| d x \leq \delta
$$

for each $t \geq \tau+T$ and $k \geq \bar{k}$.

Remark 3.1. According to Lemma 3.1, each globally defined solution $p$ of problem (2.1)-(2.3) on $[\tau, \infty)$ with $\tau \geq 0,0 \leq \varepsilon \ll 1$, and $p(\tau) \in H$, belongs to $L^{\infty}\left(\tau, \infty ; \bar{L}^{1}\right)$. In particular, the following equality holds:

$$
\mathcal{D}_{\tau, \varepsilon}^{+}=\left\{p \in \mathcal{K}_{\tau, \varepsilon}^{+}: p(\tau) \in H\right\} .
$$

The following result guaranties existence and dissipativity for the problem (2.1)-(2.4).

Theorem 3.1. Let $0<\varepsilon \ll 1$. Then for every $p_{\tau} \in H$ problem (2.1)-(2.4), (2.7) on $[\tau, T]$ has a unique solution $p$. Moreover, $p \in C([\tau, T] ; H)$. Moreover, there exists $R_{0}>0$ such that for an arbitrary bounded (in $L^{2}$ ) set $K \subset H$ and for arbitrary $\varepsilon \in(0,1)$ there exists a moment of time $T=T(K, \varepsilon)$ such that for every $\tau \geq 0$ and $p \in \mathcal{D}_{\tau, \varepsilon}^{+}$satisfying $p(\tau) \in K$ the following inequality holds:

$$
\|p(t)\|_{L^{2}} \leq R_{0}
$$

for each $t \geq \tau+T$.

The next result guaranties the continuous properties of solutions of (2.1)-(2.4).

Theorem 3.2. [14, Lemma 3.3] Let $0 \leq \tau<T<\infty, p_{\tau}^{n} \in H, b_{n} \in L^{\infty}(\tau, T)$, and $0<\varepsilon_{n} \ll 1$ for each $n=0,1, \ldots$. Suppose that $\left|b_{n}(t)\right| \leq B$ for a.e. $t \in(\tau, T)$ and $p^{n} \in C\left([\tau, T] ; H_{w}\right)$ be a solution of problem (2.1)-(2.4), (2.7) on $[\tau, T]$ with parameters $p_{\tau}^{n}, \varepsilon_{n}, b_{n}$, for each $n \geq 1$. If

$$
p_{\tau}^{n} \rightarrow p_{\tau}^{0} \text { in } H_{w}, \varepsilon_{n} \rightarrow \varepsilon_{0}>0, b_{n} \rightarrow b_{0} \text { weakly-star in } L^{\infty}(\tau, T),
$$

then there exists a solution $p \in C\left([\tau, T] ; H_{w}\right)$ of problem (2.1)-(2.4), (2.7) on $[\tau, T]$ with parameters $p_{\tau}^{0}, \varepsilon_{0}, b_{0}$, such that up to a subsequence the following convergence holds:

$$
p^{n} \rightarrow p \text { in } C\left([\tau, T] ; H_{w}\right) .
$$

Moreover, if $p_{\tau}^{n} \rightarrow p_{\tau}^{0}$ in $H$, then the following statements hold: 
(a) $p, p^{n} \in C([\tau, T] ; H)$ for each $n \geq 1$;

(b) the following convergence holds for the entire sequence:

$$
\begin{aligned}
& p^{n} \rightarrow p \text { in } L^{2}\left(\tau, T ; H^{1}\right), \\
& p^{n} \rightarrow p \text { in } C([\tau, T] ; H) .
\end{aligned}
$$

If, additionally, $b_{n} \rightarrow b_{0}$ in the Lebesgue measure on $[\tau, T]$, then

$$
\frac{\partial p^{n}}{\partial t} \rightarrow \frac{\partial p}{\partial t} \text { in } L^{2}\left(\tau, T ; H^{-1}\right)
$$

\section{Existence and properties of uniform global attractors in the non-autonomous case}

To characterize the uniform long-time behavior of solutions for non-autonomous dissipative dynamical system consider the united trajectory space $\mathcal{K}_{\varepsilon, \cup}^{+}$for the family of solutions $\left\{\mathcal{K}_{\varepsilon, \tau}^{+}\right\}_{\tau \geq 0}$ shifted to zero:

$$
\mathcal{K}_{\varepsilon, \cup}^{+}:=\bigcup_{\tau \geq 0}\left\{T(h) y(\cdot+\tau): y(\cdot) \in \mathcal{K}_{\varepsilon, \tau}^{+}, h \geq 0\right\},
$$

and the extended united trajectory space for the family $\left\{\mathcal{K}_{\varepsilon, \tau}^{+}\right\}_{\tau \geq 0}$ :

$$
\mathcal{K}_{\varepsilon}^{+}:=\operatorname{cl}_{C^{\operatorname{loc}\left(\mathbb{R}_{+} ; H\right)}}\left[\mathcal{K}_{\varepsilon, \cup}^{+}\right],
$$

where $\operatorname{cl}_{C^{\text {loc }}\left(\mathbb{R}_{+} ; H\right)}[\cdot]$ is the closure in $C^{\text {loc }}\left(\mathbb{R}_{+} ; H\right)$. Since $T(h) \mathcal{K}_{\varepsilon, \cup}^{+} \subseteq \mathcal{K}_{\varepsilon, \cup}^{+}$for each $h \geq 0$, then

$$
T(h) \mathcal{K}_{\varepsilon}^{+} \subseteq \mathcal{K}_{\varepsilon}^{+} \text {for each } h \geq 0,
$$

due to

$$
\rho_{C^{\mathrm{loc}}\left(\mathbb{R}_{+} ; H\right)}(T(h) u, T(h) v) \leq \rho_{C^{\mathrm{loc}}\left(\mathbb{R}_{+} ; H\right)}(u, v) \text { for each } u, v \in C^{\mathrm{loc}}\left(\mathbb{R}_{+} ; H\right),
$$

where $\rho_{C^{\text {loc }}\left(\mathbb{R}_{+} ; H\right)}$ is the standard metric on Fréchet space $C^{\text {loc }}\left(\mathbb{R}_{+} ; H\right)$. Therefore the set

$$
\mathbb{X}:=\left\{y(0): y \in \mathcal{K}_{\varepsilon}^{+}\right\}
$$

is closed in $H$. We endow this set $\mathbb{X}$ with metric

$$
\rho_{\mathbb{X}}\left(x_{1}, x_{2}\right)=\left\|x_{1}-x_{2}\right\|_{X}, \quad x_{1}, x_{2} \in \mathbb{X} .
$$

Then we obtain that $(\mathbb{X}, \rho)$ is a Polish space (complete separable metric space).

Let us define the multivalued semiflow (m-semiflow) $V_{\varepsilon}: \mathbb{R}_{+} \times \mathbb{X} \rightarrow 2^{\mathbb{X}}$ :

$$
V_{\varepsilon}\left(t, y_{0}\right):=\left\{y(t): y(\cdot) \in \mathcal{K}_{\varepsilon}^{+} \text {and } y(0)=y_{0}\right\}, \quad t \geq 0, y_{0} \in \mathbb{X} .
$$

According to (4.3) and (4.4) for each $t \geq 0$ and $y_{0} \in \mathbb{X}$ the set $V_{\varepsilon}\left(t, y_{0}\right)$ is nonempty. Moreover, the following two conditions hold: 
(i) $V_{\varepsilon}(0, \cdot)=I$ is the identity map;

(ii) $V_{\varepsilon}\left(t_{1}+t_{2}, y_{0}\right) \subseteq V_{\varepsilon}\left(t_{1}, V_{\varepsilon}\left(t_{2}, y_{0}\right)\right), \forall t_{1}, t_{2} \in \mathbb{R}_{+}, \forall y_{0} \in \mathbb{X}$,

where $V_{\varepsilon}(t, D)=\cup_{y \in D} V_{\varepsilon}(t, y), D \subseteq \mathbb{X}$.

We denote by $\operatorname{dist}_{\mathbb{X}}(C, D)=\sup _{c \in C} \inf _{d \in D} \rho_{\mathbb{X}}(c, d)$ the Hausdorff semidistance between nonempty subsets $C$ and $D$ of the Polish space $\mathbb{X}$. Recall that the compact set $\Theta_{\varepsilon} \subset \mathbb{X}$ is a global attractor of the m-semiflow $V_{\varepsilon}$ if it satisfies the following conditions:

(i) $\Theta_{\varepsilon}$ attracts each bounded subset $B \subset \mathbb{X}$, i.e.

$$
\operatorname{dist}_{\mathbb{X}}\left(V_{\varepsilon}(t, B), \Theta_{\varepsilon}\right) \rightarrow 0, \quad t \rightarrow+\infty ;
$$

(ii) $\Theta_{\varepsilon}$ is negatively semi-invariant set, that is, $\Theta_{\varepsilon} \subseteq V_{\varepsilon}\left(t, \Theta_{\varepsilon}\right)$ for each $t \geq 0$.

In this paper we examine the uniform long-time behavior of solution sets $\left\{\mathcal{K}_{\tau, \varepsilon}^{+}\right\}_{\tau \geq 0}$ in the strong topology of the natural phase space $H$ (as time $t \rightarrow+\infty$ for a fixed $\varepsilon>0$ ) in the sense of the existence of a compact global attractor for m-semiflow $V_{\varepsilon}$ generated by the family of solution sets $\left\{\mathcal{K}_{\tau, \varepsilon}^{+}\right\}_{\tau \geq 0}$ and their shifts.

Theorem 4.1. For each $\varepsilon>0$ the m-semiflow (4.5) has the connected stable global attractor $\Theta_{\varepsilon}$ in the phase space $\mathbb{X}$. Moreover, $\Theta_{\varepsilon}$ is bounded in $H$ uniformly in $\varepsilon$.

Proof. Due to Theorems 3.1, 3.2 and classical results about existence of global attractors (see [21]) it is sufficient to prove that $V_{\varepsilon}$ is asymptotically compact, that is,

$$
\text { every sequence }\left\{\bar{\xi}_{n} \in V_{\varepsilon}\left(t_{n}, p_{0}^{n}\right)\right\} \text { is precompact in } H \text {, }
$$

where $t_{n} \nearrow+\infty,\left\|p_{0}^{n}\right\|_{X} \leq r$.

Let $\bar{\xi}_{n} \in V_{\varepsilon}\left(t_{n}, p_{0}^{n}\right)$. Then $\exists \xi_{n}:\left\|\xi_{n}-\bar{\xi}_{n}\right\|_{\mathbb{X}}<\frac{1}{n}$ and $\xi_{n}=p_{n}\left(t_{n}\right), p_{n}$ is a solution of $(2.1)-(2.4)$ with $p_{n}(0)=p_{0}^{n}$ and $b_{n}(\cdot):=b\left(\cdot+\tau_{n}\right), \tau_{n} \geq 0$. Therefore, from Theorem 3.1

$$
\left\|p_{n}(t)\right\|_{X} \leq R_{0}+r, \forall n \geq 1, t \geq 0 .
$$

So we can claim that $\left\{\xi_{n}\right\}$ is precompact in $H_{w}$. Indeed, since $\left\|\xi_{n}\right\|_{L^{2}} \leq R_{0}+r$ then up to subsequence $\xi_{n} \rightarrow \xi$ in $L_{w}^{2}$. Let us prove that up to a subsequence $\xi_{n} \rightarrow \xi$ in $\bar{L}_{w}^{1}$. Since $\xi_{n}=p_{n}\left(t_{n}\right)$, then (3.2) yields that for each $\delta>0$ there exist $k(\delta) \geq 1, n(\delta) \geq 1$ such that

$$
\int_{|x|>k} \xi_{n}(x)|x| d x<\frac{\delta}{3}, \forall k \geq k(\delta), n \geq n(\delta) .
$$

According to Amigó et al. [1, Lemma 6.1]

$$
\left(\overline{L^{1}}\right)^{*}=\left\{\varphi=(1+|x|) u: u \in L^{\infty}\right\} .
$$


Thus, we set $d_{n}(x)=(1+|x|) \xi_{n}(x)$ and prove that $\left\{d_{n}\right\}$ is a Cauchy sequence in $L_{w}^{1}$, because

$$
\begin{aligned}
& \left|\int_{\mathbb{R}}\left(d_{n}(x)-d_{m}(x)\right) u(x) d x\right| \leq\left|\int_{|x| \leq k}(1+|x|)\left(\xi_{n}(x)-\xi_{m}(x)\right) u(x) d x\right| \\
& +2\|u\|_{L^{\infty}}\left(\int_{|x|>k} \xi_{n}(x)|x| d x+\int_{|x|>k} \xi_{m}(x)|x| d x\right)<\delta
\end{aligned}
$$

for each $u \in L^{\infty}$ and $n, m \geq N=N(\delta, k)$. Since the space $L^{1}$ is weakly complete, then up to a subsequence $\bar{d}_{n} \rightarrow d$ in $L_{w}^{1}$ for some $d \in L^{1}$. Thus

$$
\xi_{n} \rightarrow \bar{\xi}=\frac{d}{1+|x|} \text { in } \bar{L}_{w}^{1} .
$$

If we consider the restriction of $\xi_{n}$ to each interval $[-k, k]$, then we deduce that $\bar{\xi}=\xi$ and up to a subsequence $\xi_{n} \rightarrow \xi$ in $H_{w}$.

Now let us prove this convergence in the strong topology of $H$. Consider a smooth real function $\theta$ that satisfies the following three conditions:

$$
\begin{array}{lll}
\text { (a) } & \theta(s)=0, & |s| \leq 1 ; \\
\text { (b) } & 0 \leq \theta(s) \leq 1, & |s| \in[1,2] \text {; } \\
\text { (c) } & \theta(s)=1, & |s| \geq 2,
\end{array}
$$

and define for $k>1$

$$
\rho_{k}(x)=\theta\left(\frac{x}{k}\right) .
$$

According to Amigó et al. [1, pp. 215-216] after multiplying (2.1) by $\rho_{k}(x) p_{n}$ we obtain

$$
\begin{aligned}
& \frac{1}{2} \frac{d}{d t} \int_{\mathbb{R}} \rho_{k}(x) p_{n}^{2} d x+b_{n}(t) \int_{\mathbb{R}} \rho_{k}(x) p_{n} \frac{\partial p_{n}}{\partial x} d x \\
& \quad+\left(D\left(p_{n}(\cdot, t)\right)+\varepsilon_{n}\right)\left(\int_{\mathbb{R}} \rho_{k}(x)\left(\frac{\partial p_{n}}{\partial x}\right)^{2} d x\right. \\
& \left.\quad+\frac{1}{k} \int_{\mathbb{R}} \theta^{\prime}\left(\frac{x}{k}\right) p_{n} \frac{\partial p_{n}}{\partial x} d x\right)+\int_{\mathbb{R}} \rho_{k}(x) p_{n}^{2} d x=0 .
\end{aligned}
$$

Integrating by parts we deduce

$$
\begin{gathered}
b_{n}(t) \int_{\mathbb{R}}\left(\rho_{k}(x) p_{n} \frac{\partial p_{n}}{\partial x} d x=-\frac{b_{n}(t)}{2 k} \int_{\mathbb{R}} \theta^{\prime}\left(\frac{x}{k}\right) p_{n}^{2} d x,\right. \\
\frac{1}{k} \int_{\mathbb{R}} \theta^{\prime}\left(\frac{x}{k}\right) p_{n} \frac{\partial p_{n}}{\partial x} d x=-\frac{1}{2 k^{2}} \int_{\mathbb{R}} \theta^{\prime \prime}\left(\frac{x}{k}\right) p_{n}^{2} d x .
\end{gathered}
$$

Then from (4.9) we have

$$
\frac{1}{2} \frac{d}{d t} \int_{\mathbb{R}} \rho_{k}(x) p_{n}^{2} d x+\int_{\mathbb{R}} \rho_{k}(x) p_{n}^{2} d x \leq\left(\frac{B \beta}{2 k}+\frac{(\alpha+1) \beta}{2 k^{2}}\right) \int_{\mathbb{R}} p_{n}^{2} d x,
$$


where $\beta:=\max _{|s| \in[1,2]}\left\{\left|\theta^{\prime}(s)\right|+\left|\theta^{\prime \prime}(s)\right|\right\}$.

Combining (4.7) and (4.10) we deduce from Gronwall's Lemma that for some positive constant $C=C(r)$

$$
\int_{|x|>2 k} p_{n}^{2}(x, t) d x \leq e^{-2 t} r^{2}+\frac{C(r)}{k}, \forall t \geq 0, n \geq 1, k>1 .
$$

On the other hand, for every solution of (2.1)-(2.4) we have the following energy equality (for details see the proof of Lemma 3.2):

$$
\begin{aligned}
\frac{1}{2} \frac{d}{d t} \int_{\mathbb{R}}(p(x, t))^{2} d x & +(D(p(\cdot, t))+\varepsilon) \int_{\mathbb{R}}\left(\frac{\partial p(x, t)}{\partial x}\right)^{2} d x+\int_{|x|>1}(p(x, t))^{2} d x \\
& =\frac{D(p(\cdot, t))}{\alpha}\left\langle\delta_{0}, p(\cdot, t)\right\rangle .
\end{aligned}
$$

Let us consider the functions

$$
\bar{p}_{n}(t)=p_{n}\left(t+\left(t_{n}-1\right)\right), t \geq 0 .
$$

Then $\bar{p}_{n}$ is a solution of (2.1)-(2.4) with $\bar{b}_{n}(\cdot):=b_{n}\left(\cdot+t_{n}-1\right)=b\left(\cdot+t_{n}-1+\tau_{n}\right)$, $\bar{p}_{n}(0)=p_{n}\left(t_{n}-1\right), \bar{p}_{n}(1)=\xi_{n}$ and $\bar{p}_{n}$ satisfies (4.7), (4.9), (4.12). Moreover, similarly to the previous arguments we deduce that up to subsequence

$$
\bar{p}_{n}(0)=p_{n}\left(t_{n}-1\right) \rightarrow \bar{p}_{0} \text { in } H_{w} .
$$

Hence, from Lemma 3.2 we obtain for every $T>1$ that

$$
\bar{p}_{n} \rightarrow \bar{p} \text { in } C\left([0, T] ; H_{w}\right),
$$

where $\bar{p}$ is a solution of (2.1)-(2.4) with $\bar{p}(0)=\bar{p}_{0}$ and some $\bar{b} \in L^{\infty}(0,+\infty)$ such that $\bar{b}_{n} \rightarrow b$ weakly star in $L^{\infty}(0, T)$ for each $T>0$. In particular, $|\bar{b}(t)| \leq B$ for a.e. $t>0$.

Since $\varepsilon>0$ is fixed, we can derive from (4.7), (4.12) and the Aubin-Lions theorem [16] that for every $k>1$ up to subsequence

$$
\bar{p}_{n} \rightarrow \bar{p} \text { in } L^{2}\left(0, T ; L^{2}(-k, k)\right) .
$$

In particular,

$$
\bar{p}_{n}(t) \rightarrow \bar{p}(t) \text { in } L^{2}(-k, k) \text { for a.a. } t \in(0, T) .
$$

By a diagonal procedure we obtain that up to a subsequence and for some $\tau \in(0,1)$,

$$
\bar{p}_{n}(\tau) \rightarrow \bar{p}(\tau) \text { in } L^{2}(-k, k), \forall k \geq 1 .
$$

From (4.11) we get

$$
\int_{|x|>2 k} \bar{p}_{n}^{2}(x, \tau) d x \leq e^{-2\left(\tau+t_{n}-1\right)} r^{2}+\frac{C(r)}{k}, \forall n \geq 1, k>1 .
$$


Combining (3.2), (4.14), (4.15) we have

$$
\bar{p}_{n}(\tau) \rightarrow \bar{p}(\tau) \text { in } X .
$$

Then the second part of Theorem 3.2 guarantees the convergence

$$
\bar{p}_{n} \rightarrow \bar{p} \text { in } C([\tau, T] ; H) .
$$

In particular,

$$
\xi_{n}=\bar{p}_{n}(1) \rightarrow \bar{p}(1) \quad \text { in } H .
$$

Thus we obtain the required precompactness of $\left\{\xi_{n}\right\}$ and, therefore, the existence of the connected, stable global attractor $\Theta_{\varepsilon}$.

\section{Acknowledgment}

The first two authors were partially supported by the National Academy of Sciences of Ukraine, Grant 2290-18. The third author was partially supported by Spanish Ministry of Economy and Competitiveness and FEDER, projects MTM2015-63723-P and MTM2016-74921-P, and by Junta de Andalucía (Spain), project P12-FQM-1492.

\section{References}

1. J. M. Amigo, I. Catto, A. Gimenez, J. Valero, Attractors for a non-linear parabolic equation modelling suspension flows, Discrete Contin. Dyn. Sist., Series B, 11(2009), 205-231.

2. J.M. Amigo, A. Gimenez, F. Morillas, J. Valero, Attractors for a lattice dynamical system generated by non-Newtonian fluids modeling suspensions, Internat. J. Bifur. Chaos Appl. Sci. Engrg., 20(2010), 2681-2700. DOI: $10.1142 / \mathrm{S} 0218127410027295$.

3. A.V. Babin, M.I. Vishik, Attractors of Evolution Equations, Nauka, Moscow, 1989.

4. E. Cances, I. CATto, Yo. Gati, Mathematical analysis of a nonlinear parabolic equation arising in the modelling of non-Newtonian flows, SIAM J. Math. Anal., 37(2005), 60-82. DOI: 10.1137/S0036141003430044.

5. E. CANCES, C. LE BRIS, Convergence to equilibrium of a multiscale model for suspensions, Discrete Contin. Dyn. Sist., Series B, 6(2006), 449-470. DOI: 10.3934/dcdsb.2006.6.449.

6. V.V. Chepyzhov, M.I. VishiK, Evolution equations and their trajectory attractors, J. Math. Pures Appl., 76(1997), 913-964. DOI: 10.1016/S00217824(97)89978-3.

7. E.A. Feinberg, P.O. Kasyanov, M.Z. Zgurovsky, Uniform Fatou's lemma, J. Math. Anal. Appl., 444(2016), 550-567. DOI: 10.1016/j.jmaa.2016.06.044.

8. H. Gajewsini, K. Groger, K. Zacharias, Nichtlineare Operatorgleichungen und Operatordifferentialgleichungen, Akademie-Verlag, Berlin, 1975. DOI: $10.1002 /$ mana. 19750672207 
9. A. Gimenez, F. Morillas, J. Valero, J.M. Amigo, Stability and numerical analysis of the Hebraud-Lequeux model for suspensions, Discrete Dyn. Nat. Soc., 2011(2011), Art. ID 415921, 24 pp.

10. N.V. Gorban, O.V. Kapustyan, P.O. Kasyanov, Uniform trajectory attractor for non-autonomous reaction-diffusion equations with Carathéodory's nonlinearity, Nonlinear Anal., 98(2014), 13-26. DOI: 10.1016/j.na.2013.12.004.

11. N.V. Gorban, O.V. Kapustyan, P.O. Kasyanov, Uniform trajectory attractor for non-autonomous reaction-diffusion equations with Caratheodory's nonlinearity, Nonlinear Analysis, 98(2014), 13-26. http://dx.doi.org/10.1016/j.na.2013.12.004.

12. P. Hebraud, F. Lequeux, Mode-coupling theory for the pasty rheology of soft glassy materials, Phys. Rev. Lett., 81(1998), 2934-2937.

13. O.V. Kapustyan, J. Valero, P.O. Kasyanov, A. Gimenez, J.M. Amigo, Convergence of numerical approximations for a non-Newtonian model of suspensions, Internat. J. Bifur. Chaos Appl. Sci. Engrg., 25(2015), 1540022, 24 pp.

14. O.V. Kapustyan, P.O. Kasyanov, J. Valero, M.Z. Zgurovsky Strong attractors for vanishing viscosity approximations of non-Newtonian suspension flows, Discrete and Continuous Dynamical Systems, 23(3)(2018), 1155-1176. DOI: $10.3934 /$ dcdsb.2018146.

15. O.A. Ladyzhenskaya, Attractors for Semigroups and Evolution Equations, Cambridge University Press, Cambridge, 1991.

16. R. Temam, Navier-Stokes Equations, North-Holland, Amsterdam, 1979.

17. R. Temam, Infinite-dimensional Dynamical Systems in Mechanics and Physics, Springer-Verlag, New York, 1988.

18. J. Valero, A. Gimenez, O. V. Kapustyan, P. Kasyanov, J. M. Amigo, Convergence of equilibria for numerical approximations of a suspension model, Comput. Math. Appl., 72(2016), 856-878. DOI: 10.1016/j.camwa.2016.05.034.

19. K. Yosida, Functional Analysis, Springer, Berlin, 1980.

20. M.Z. Zgurovsky, P.O. Kasyanov, J. Valero, Noncoercive evolution inclusions for Sk type operators, Internat. J. Bifur. Chaos Appl. Sci. Engrg., 20(2010), 28232834. DOI: $10.1142 /$ S0218127410027386.

21. M.Z. Zgurovsky, P.O. Kasyanov, O.V. Kapustyan, J. Valero, N.V. ZADOIANCHUK, Evolution Inclusions and variation inequalities for earth data processing III, Springer-Verlag, Berlin, 2012.

22. M.Z. Zgurovsky, P.O. Kasyanov, Multivalued dynamics of solutions for autonomous operator differential equations in strongest topologies, Continuous and Distributed Systems: Theory and Applications. Solid Mechanics and Its Applications, 211(2014), 149-162.

23. M.Z. Zgurovsky, P.O. Kasyanov, Evolution Inclusions in Nonsmooth Systems with Applications for Earth Data Processing : Uniform Trajectory Attractors for Nonautonomous Evolution Inclusions Solutions with Pointwise Pseudomonotone Mappings, Advances in Global Optimization, Springer Proceedings in Mathematics \& Statistics, 95(2015), 283-294. DOI: 10.1007/978-3-319-08377-3_28.

24. M.Z. Zgurovsky, P.O. Kasyanov, Uniform Trajectory Attractors for Nonautonomous Dissipative Dynamical Systems, Continuous and Distributed Systems II. Series Studies in Systems, Decision and Control, 30(2015), 221-232. DOI: 10.1007/978-3-319-19075-4_13.

25. M.Z. Zgurovsky, P.O. KAsyanov, Uniform Global Attractors for Nonautonomous Evolution Inclusions, Advances in Dynamical Systems and 
Control. Studies in Systems, Decision and Control, 69(2016). DOI: 10.1007/978-3319-40673-2 3 .

26. M.Z. Zgurovsky, M.O. Gluzman, N.V. Gorban, L.S. Paliichuk, O.V. Khomenko, Uniform Global Attractors for Non-Autonomous Dissipative Dynamical Systems, Discrete and Continuous Dynamical Systems, 22(5)(2017), 2053-2065. DOI: 10.3934/dcdsb.2017120. 